\title{
Is Endoscopic Band Ligation a Superior Treatment Modality for Gastric Antral Vascular Ectasia Compared to Argon Plasma Coagulation?
}

\author{
Neil Robert O'Morain, Helen O'Donovan, Caroline Conlon, Eileen Shannon, Diarmuid Manning and Eoin Slattery \\ Department of Gastroenterology, University Hospital Galway, Galway, Ireland
}

Background/Aims: Gastric antral vascular ectasia (GAVE) is a rare acquired vascular lesion of the gastric antrum. The most frequent presentation of GAVE is iron deficiency anemia. Endoscopic therapy is the mainstay of treatment. However, there is no consensus regarding the optimal treatment modality.

Methods: A retrospective cohort study was performed on patients with GAVE, including patients receiving endoscopic therapy. Treatment was with either argon plasma coagulation (APC) or endoscopic band ligation (EBL). Basic demographic data, indication for index procedure, number of sessions, and pre- and post-hemoglobin levels were collected. The aim of the study was to compare outcomes across the two treatment modalities.

Results: One hundred and seventeen diagnoses of GAVE were made. Sixty-two patients (53\%) required endoscopic treatment for symptomatic GAVE (female, $n=38,61 \%$; mean age of 74.4 years). Two hundred and eighteen procedures were performed during the study period. APC was performed $(n=161,74 \%)$ more frequently than EBL $(n=57,26 \%)$. Patients treated with APC at index required a median 5 subsequent therapeutic interventions (APC or EBL), while those treated with EBL at index required a further 2.9 treatments (EBL only) $(p<0.05)$.

Conclusions: APC was the most common treatment modality employed. We demonstrate an increasing incidence of EBL. Patients treated with EBL at index treatment required fewer subsequent treatment sessions and had a greater mean rise in hemoglobin. This suggests a more effective endoscopic response with EBL. Clin Endosc 2021;54:548-554

Key Words: Anemia, iron-deficiency; Argon plasma coagulation; Endoscopic band ligation; Gastric antral vascular ectasia; Retrospective study

\section{INTRODUCTION}

Gastric antral vascular ectasia (GAVE) is an infrequently encountered vascular lesion located primarily in the gastric antrum. It accounts for approximately $4 \%$ of cases of non-variceal upper gastrointestinal bleeding. It is characterised by a

Received: August 24, 2020 Revised: October 13, 2020

Accepted: October 13, 2020

Correspondence: Neil Robert O’Morain

Department of Gastroenterology, University Hospital Galway, Newcastle Road, Galway H91 YR71, Ireland

Tel: +353-86-104-8627, Fax: +353-09-152-4222, E-mail: neilomorain@svhg.ie

ORCID: https://orcid.org/0000-0003-2542-9455

(c) This is an Open Access article distributed under the terms of the Creative Commons Attribution Non-Commercial License (http://creativecommons.org/ licenses/by-nc/3.0) which permits unrestricted non-commercial use, distribution, and reproduction in any medium, provided the original work is properly cited. distinctive endoscopic appearance consisting of visible convoluted vessels which may present in a diffuse disorganised and nodular pattern located in the antrum, or in prominent organised streaks and stripes emanating from the pylorus resembling the stripes of a watermelon (Fig. 1). Endoscopic description of extent and severity is largely subjective, with the decision to treat and the selection of treatment modality generally based on endoscopists' preference. While histological diagnosis is rarely required, biopsies show dilated, ectatic capillaries in the mucosa and submucosa with microthrombi and minimal inflammation. ${ }^{1,2}$

GAVE was first described as an arteriovenous malformation of the stomach and treated by antrectomy with Billroth-I anastomosis formation, which while resulting in an improvement in hemoglobin levels, resulted in significant morbidity. ${ }^{3,4}$ Early endoscopic therapies included heat probe, ${ }^{5}$ bipolar electroco- 


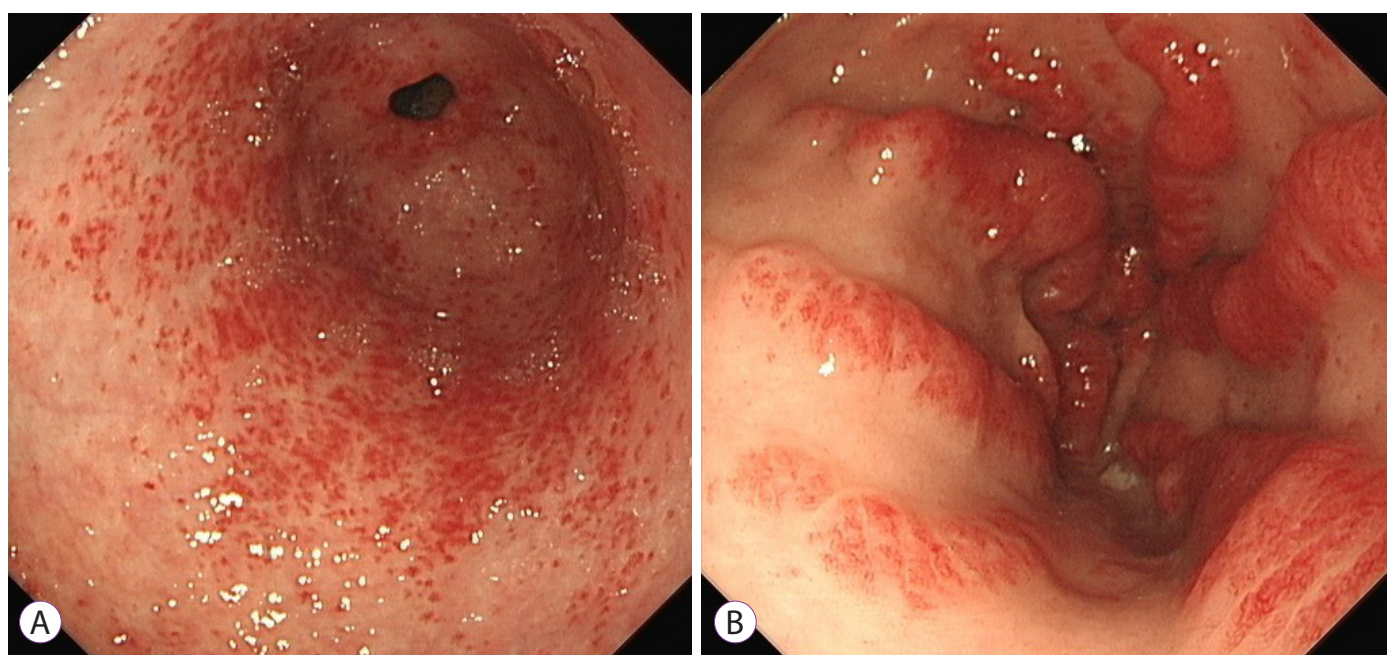

Fig. 1. Endoscopic appearance of gastric antral vascular ectasia; diffuse disorganised and nodular pattern located in the antrum (A), or in prominent organised streaks and stripes emanating from the pylorus resembling the stripes of a watermelon (B).
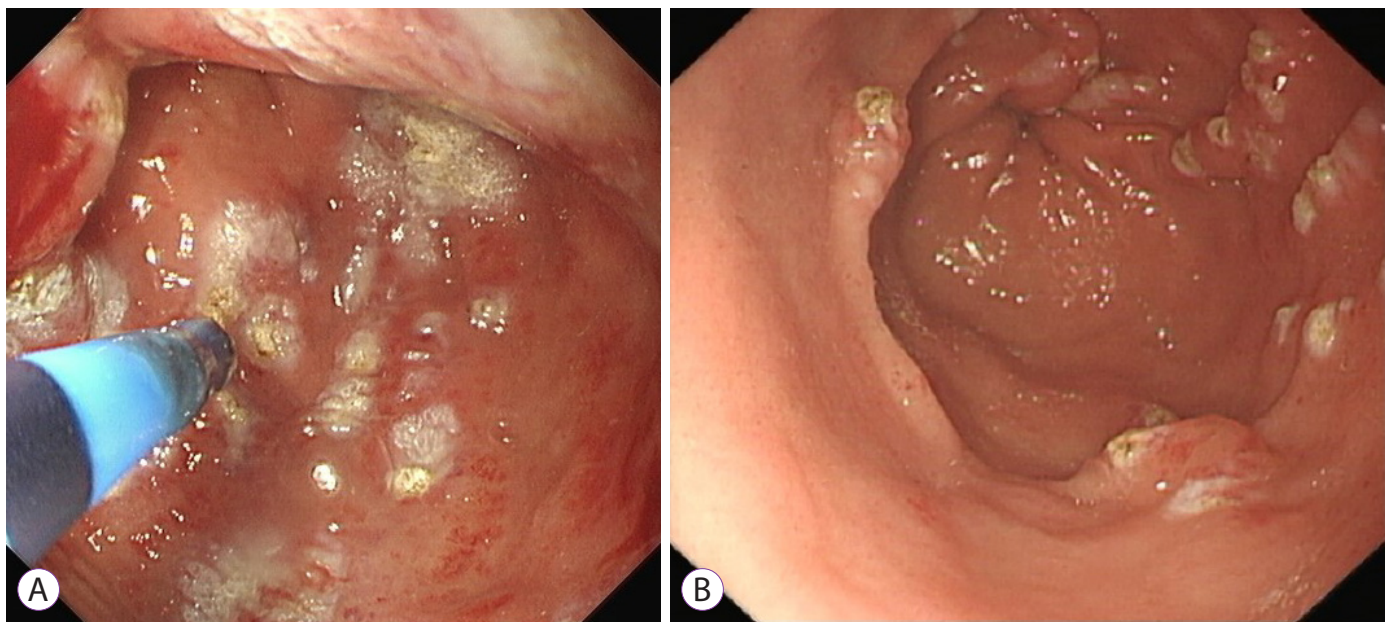

Fig. 2. (A, B) Treatment of gastric antral vascular ectasia with argon plasma coagulation.

agulation, ${ }^{6}$ sclerotherapy, ${ }^{7}$ and laser therapy. ${ }^{8}$ These were initially effective endoscopic modalities but were limited by cost, availability, and the risk of deep thermal injury. Argon plasma coagulation (APC), which induces coagulation of the tissue surface using a high-frequency electrical current conducted via ionised argon gas, has become the treatment modality of choice for most endoscopists as it is easy to use, relatively safe, and familiar to endoscopists (Fig. 2). The depth of penetration usually ranges from $1-3 \mathrm{~mm}$, significantly reducing the risk of perforation. Initial reports demonstrated an $80 \%$ success rate in reducing transfusion requirements, ${ }^{9,10}$ for both the diffuse-type and the stripe-type of GAVE ${ }^{11}$; however, it often required multiple treatment sessions. ${ }^{12}$ More recent studies have highlighted the limitations of APC on follow-up, with higher treatment failures, and only a small number of patients remaining transfusion free post treatment. ${ }^{13-16}$ Radiofrequency ablation (RFA), which transmits high-power energy in short bursts to ablate superficial mucosal lesions, has been proposed as an alternative to APC, particularly for treatment-refractory cases. ${ }^{1718}$ However, there are few prospective trials investigating RFA in GAVE with sparse data on long-term outcomes.

Endoscopic band ligation (EBL) has become an increasingly used alternative treatment modality, ${ }^{19}$ demonstrating a good safety profile and favourable effectiveness. ${ }^{20} \mathrm{~A}$ clinical response of $>90 \%$ has been reported on short term follow-up. ${ }^{21}$ Given that the vascular lesions observed in GAVE often involve the submucosa, the deeper penetration offered by band ligation should result in more effective hemostasis (Fig. 3). Recent 


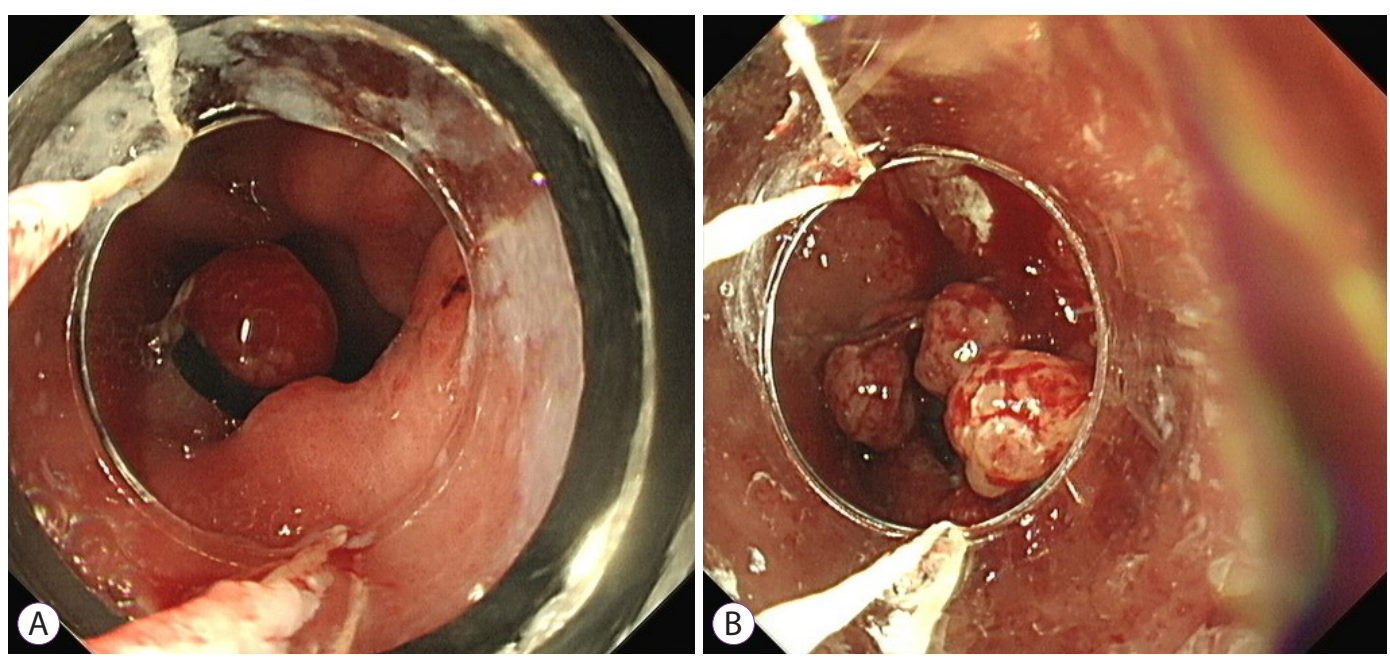

Fig. 3. (A, B) Treatment of gastric antral vascular ectasia with endoscopic band ligation.

studies demonstrating lower re-bleeding rates following EBL as compared to APC, and higher treatment success rates would support this. ${ }^{19,22}$ However, a recent retrospective study demonstrated a high initial rate of clinical response with falling recurrence-free survival over time, suggesting the longterm outcomes may not be as successful as proposed. ${ }^{23}$

While an increasing number of treatment options are being explored for GAVE, currently no evidence-based treatment algorithm exists to direct the appropriate treatment approach for GAVE. It remains unknown whether certain sub-types of GAVE respond better to particular treatment modalities, or whether treatment could be tailored or indeed, individualised.

The aim of this study was to retrospectively evaluate the clinical course of patients treated with either APC or EBL for GAVE to determine whether either one conferred better longterm outcomes.

\section{MATERIALS AND METHODS}

\section{Patient selection}

A retrospective cohort study was performed on all patients with an endoscopic diagnosis of GAVE (ICD-9 code 537.82) since the adoption of electronic records at our tertiary referral university hospital (2013-2018). Patients classified as moderate to severe GAVE (high burden of endoscopic lesions +/significant anemia/symptoms) who required endoscopic therapy were included in the study, while those considered to have mild GAVE (low burden of endoscopic lesions, borderline anemia), or not requiring endoscopic therapy were excluded. Treatment groups were divided by index treatment modality into either APC or EBL groups.

\section{Treatment modality selection}

The choice between treatment modalities was based on the endoscopists' preference and expertise. The total number of therapeutic procedures performed during the study period, as well as the index treatment for each individual patient, was recorded. Subsequent therapeutic interventions, if required, were registered. The efficacy of each treatment modality was evaluated based on: (1) requirement for repeat endoscopic therapy (i.e., persistence of GAVE/recurrence of anemia), (2) change in hemoglobin levels pre and post procedure, and (3) reduction in blood transfusion requirement. Due to the absence of a treatment algorithm and the relative novelty of EBL, several patients received crossover treatment (i.e., APC first followed by EBL). All patients treated initially with EBL were subsequently followed only with EBL. Patients were therefore divided into three groups following index treatment: APC as index followed by APC alone (group 1), APC as index followed by crossover treatment (group 2), and EBL as index followed by EBL alone (group 3).

\section{Outcomes}

The primary outcome of the study was to evaluate the comparative effectiveness of APC and EBL in the treatment of GAVE. This was measured by comparing outcomes following the index treatment (APC vs. EBL). The surrogate measures of effectiveness evaluated were the number of subsequent treatment sessions required, the rise in hemoglobin levels post procedure, and reduction in blood transfusion requirements. 


\section{Surrogate measures}

Basic demographic data and indication for index procedure were recorded. The choice of index treatment modality was documented as well as the number of treatment sessions required. Baseline and follow-up hemoglobin levels were collected. Pre-procedure level (baseline) was considered the most recent hemoglobin (up to one month) level measured prior to the intervention and post-procedure level (follow-up) was measured at an interval of 3 months following the intervention. Global transfusion requirements for the APC (groups 1 \& 2) and EBL (group 3) cohorts were recorded pre and post procedure using patient transfusion records in terms of total units of packed red cells for each group.

\section{Statistical analysis}

Data are presented as means with ranges for demographic variables. Statistical comparisons between treatment groups were made using unpaired student $t$-tests of means. A $p$-value $<0.05$ was considered statistically significant.

\section{RESULTS}

\section{Patient characteristics}

A total of 117 cases of GAVE were identified during the study period. Of these, 55 (47\%) were classified as mild GAVE and did not require endoscopic treatment. These were there- fore excluded from further analysis. The remaining 62 (53\%) moderate to severe cases were treated endoscopically with either APC or EBL (Fig. 4). Those requiring treatment displayed a female preponderance $(n=38,61 \%)$ and a mean age of 74.4 years (range, 45-95). Patient demographics are presented in Table 1. Mean follow-up period was 19.9 months (range, 2-55).

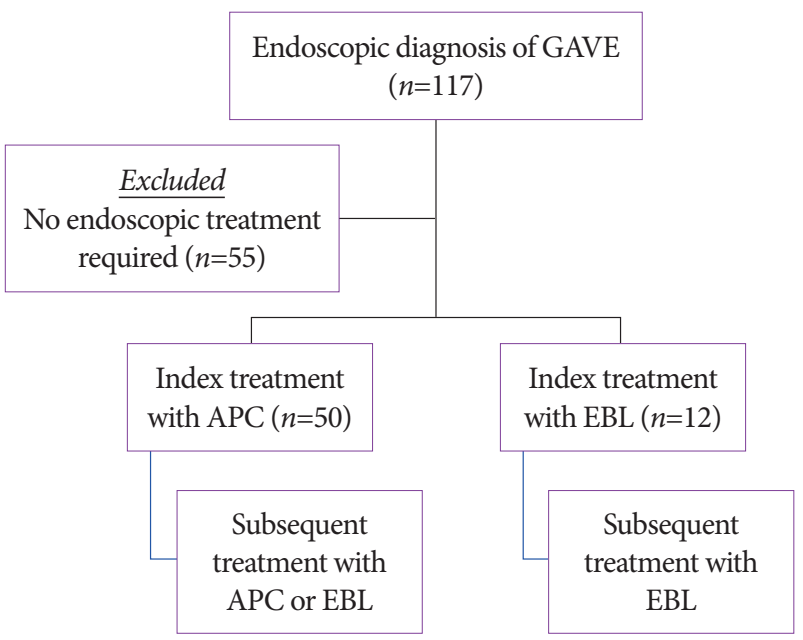

Fig. 4. Flowchart of included/excluded patients and index treatment modality. APC, argon plasma coagulation; EBL, endoscopic band ligation; GAVE, gastric antral vascular ectasia.

Table 1. Patient Demographics

\begin{tabular}{lcc}
\hline Demographics & APC group & EBL group \\
\hline Age, yr (median, IQR) & $73(70-79)$ & $75(70-78)$ \\
Sex & $22(40 \%)$ & $4(33 \%)$ \\
Male & $30(60 \%)$ & $8(66 \%)$ \\
Female & & $12(20 \%)$ \\
Treatment & $50(80 \%)$ & 56 \\
Index treatment with APC & 162 & $1.7 \mathrm{~g} / \mathrm{dL}$ \\
Index treatment with EBL & $1 \mathrm{~g} / \mathrm{dL}$ & $3(25 \%)$ \\
Total procedures & & $2(16 \%)$ \\
Mean rise in hemoglobin $(3$ months post-procedure) & $10(20 \%)$ & $1(8 \%)$ \\
Associated conditions & $8(16 \%)$ & $2(16 \%)$ \\
Cirrhosis & $11(22 \%)$ & $8(20 \%)$ \\
Chronic kidney disease & & \\
Hypothyroidism & & \\
Connective tissue disease & & \\
\hline
\end{tabular}

APC, argon plasma coagulation; EBL, endoscopic band ligation; IQR, interquartile range. 


\section{Endoscopic treatment}

Indications for index endoscopy included iron deficiency anemia $(71 \%)$, melena (13\%), variceal assessment (3\%), hematemesis (4\%) and others (9\%) (Fig. 5). In total, 218 therapeutic procedures (APC or EBL) were recorded for the treatment of GAVE during the study period. APC was the most common procedure employed by the endoscopist $(n=161$, $74 \%)$ compared with EBL ( $n=57,26 \%)$.

In terms of index treatment, APC was the most frequently selected therapeutic modality $(n=50,83 \%)$ compared with EBL $(n=12,17 \%)$ (Fig. 4). Patients who had received EBL as their index treatment continued to be treated with EBL. Those

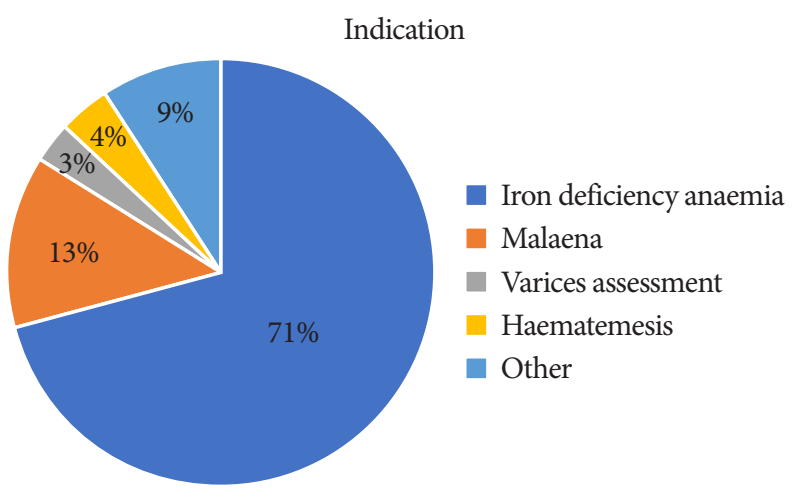

Fig. 5. Indication for upper gastrointestinal endoscopy in patients treated for moderate to severe gastric antral vascular ectasia.

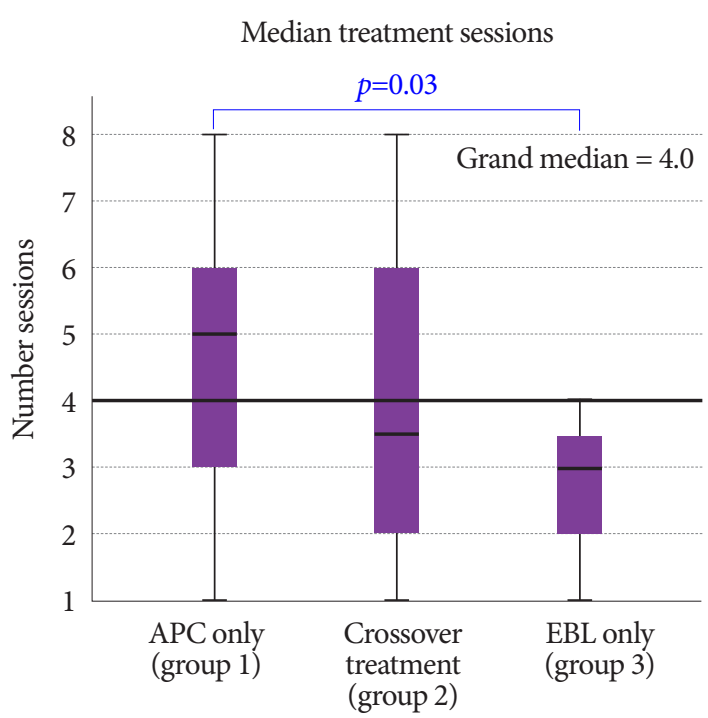

Fig. 6. Treatment sessions following index treatment $(n=63)$. APC, argon plasma coagulation; EBL, endoscopic band ligation. who had been treated initially with APC received a combination of APC and/or EBL ( $n=13)$ as subsequent treatment modalities.

\section{Endoscopic response}

Patients who had been treated with APC as the index treatment required a median of 5 subsequent therapeutic interventions when treated by APC alone $(n=37)$ and 3.5 subsequent therapeutic interventions on crossover treatment with APC or EBL $(n=13)$. Meanwhile, those treated with EBL at index $(n=12)$ required a further 2.9 treatments (EBL only) $(p=0.03)$ (Fig. 6). Both interventions resulted in a mean rise in hemoglobin levels from baseline. The APC group demonstrated a mean rise of $1.15 \mathrm{~g} / \mathrm{dL}(9.21 \mathrm{~g} / \mathrm{dL} \rightarrow 10.36 \mathrm{~g} / \mathrm{dL})(p \leq 0.05)$ while the EBL group also demonstrated a significant mean rise of $1.6 \mathrm{~g} / \mathrm{dL}(8.94 \mathrm{~g} / \mathrm{dL} \rightarrow 10.52 \mathrm{~g} / \mathrm{dL})(p \leq 0.05)$. Of note, those that received $\mathrm{APC}$ as an index treatment and subsequent treatment with EBL also demonstrated a mean rise in hemoglobin of $1.9 \mathrm{~g} / \mathrm{dL}(8.7 \mathrm{~g} / \mathrm{dL} \rightarrow 10.6 \mathrm{~g} / \mathrm{dL})(p=0.06)$. There was a more significant overall reduction in blood transfusion requirements in the EBL group post treatment compared to the APC group (reduction of $55 \%$ as compared to $15 \%$ ) (Fig. 7).

\section{Complications}

There were no significant complications in either the APC or EBL group. Post-procedure pain was reported in $16 \%(n=2)$ in the EBL group which was managed with simple analgesia.
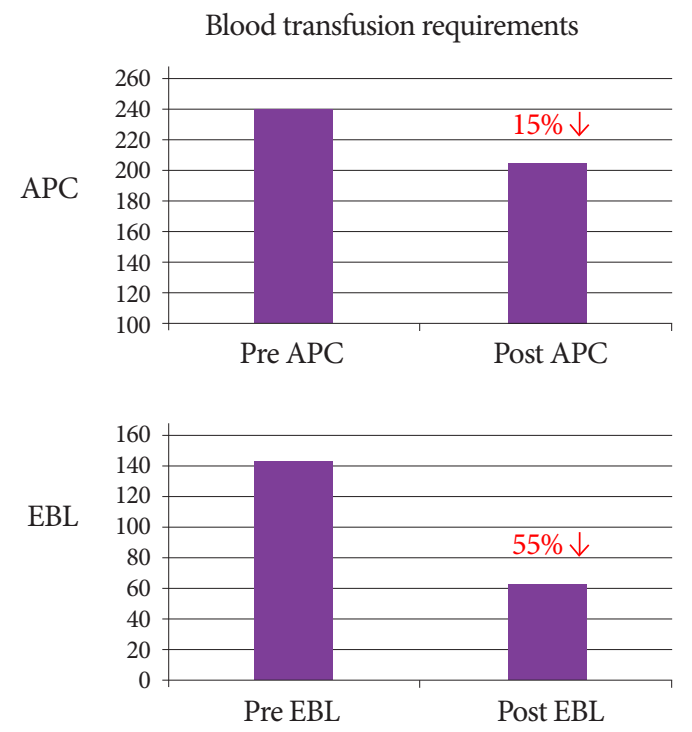

Fig. 7. Blood transfusion requirements (units) per group pre and post intervention. APC, argon plasma coagulation; EBL, endoscopic band ligation. 


\section{DISCUSSION}

This study evaluated the comparative effectiveness of APC and EBL as treatment modalities for GAVE. Using a retrospective approach, we tracked outcomes using the index treatment modality as a starting point. The decision to treat and the choice of therapy were based on the endoscopists' preference in real time, with experience the only influencing factor. The median follow-up period for the cohort was 19.9 months (range, 2-55).

The precise goal of treatment in GAVE is an interesting subject and one that lacks consensus-whether total eradication of GAVE is necessary, or whether control of bleeding by way of stable hemoglobin/iron levels is sufficient. As a retrospective study, we aimed to evaluate clinical outcomes based on the need for repeat procedure/intervention, hemoglobin levels post procedure, and transfusion requirements. In that regard, treatment outcomes were based on the more conservative measure of evidence of bleeding. Subsequent treatment sessions were booked based on the endoscopists' subjective assessment of necessity.

In our centre, as in most, APC remains the default treatment of choice in the treatment in GAVE. This is largely due endoscopists' experience with this modality in other settings such as in the treatment of radiation proctitis and angiodysplasia. It is familiar to both endoscopists and endoscopy nurses, relatively easy to use, and provides immediate results. The applied mucosal injury gives a satisfactory visual result; however, the superficial nature of the therapy leads to significant rates of recurrence. As a result, alternative therapeutic modalities have been explored.

As a relatively novel treatment modality, the incidence of EBL use is comparatively low in our cohort and its use was limited in the early stages of the study period. However, we note that its use increased during the study period, with patients initially treated with APC receiving crossover treatment with EBL. The ability of this modality to penetrate deeper into the submucosa, with resulting and subsequent thrombosis, underlies its potential as a more effective therapeutic option. ${ }^{24}$ As endoscopists become more familiar with this treatment option, the frequency of its use will likely rise further.

Our data suggests a more effective endoscopic response with EBL compared to APC, with fewer subsequent treatment sessions required and a lower transfusion requirement post treatment. This analysis includes those treated with primary EBL and those treated with 'rescue' EBL, or EBL after treatment failure with APC. The analysis in the rescue group failed to reach statistical significance likely due to the small number of patients involved in this subgroup. Blood transfusion requirements were noted to be lower 3 months post procedure in the EBL group when compared to the APC group. This crude data looked at overall transfusion requirements for the EBL and APC groups and did not correct for potential bias. As we posit that more severe cases were selected for treatment with EBL (this is supported by the lower mean hemoglobin level observed in the EBL group pre-treatment), any bias would likely inflate the pre-procedure transfusion requirement in this group. The overall reduction (reduction of 55\% in EBL compared to $15 \%$ in APC), while crude, demonstrates an important difference between the groups.

In our cohort, patients with cirrhosis and GAVE were less represented than in some earlier studies. Nonetheless, the proportion of associated conditions reported are similar to those in recent studies assessing therapeutic intervention in GAVE. ${ }^{18}$ The lower proportion of cirrhosis in our study may be explained by: (1) over-diagnosis of GAVE in cirrhotic patients with portal hypertensive gastropathy, and (2) cirrhotic patients with GAVE may represent a milder form and were not considered for therapy.

The main limitation of this study is its retrospective nature. Patients were not randomised to a particular treatment modality. In spite of this, the data we present does demonstrate a clear difference in outcomes between the two groups and presents EBL as a more effective treatment modality.

There is currently no consensus or definition of severity of disease in GAVE, or indeed, evidence to suggest whether different subtypes of disease (i.e., diffuse vs. striped) would benefit from particular treatment modalities. We suggest that cases receiving endoscopic therapy by definition represent moderate to severe GAVE; however, this is based on a subjective assessment by the endoscopist. In the absence of evidence-based treatment algorithms, the decision to treat endoscopically as well as the choice of therapy is based on endoscopist's experience and preference. This may result in inconsistent approaches to the management of GAVE and/or inappropriate or ineffective therapeutic interventions.

In our cohort, with a mean age of 74 years and a female preponderance, APC was the commonest treatment modality employed; however, we demonstrated an increasing incidence of EBL. As a relatively new treatment modality, the incidence of EBL as an index treatment was perhaps disappointingly low in our cohort. However, we do note an increase in its use as a rescue therapy for patients who are refractory to APC. Patients treated with EBL at their index treatment required fewer subsequent treatment sessions, had a greater mean rise in hemoglobin, and had a lower transfusion requirement post treatment. This data suggests a more effective endoscopic response when EBL is employed in the treatment of GAVE, either as a primary treatment modality or after APC failure. Whether certain GAVE subtypes may benefit from different treatment 
modalities is beyond the scope of this study but represents an appealing future research question. We suggest in our study that more severe cases of GAVE may be preferentially selected for treatment with EBL as more diffuse and severe presentations may respond better to the deeper penetration of EBL compared to APC. Differentiating between GAVE subtypes may facilitate the development of an evidence-based treatment algorithm which could be employed to select which patients require treatment as well as the most appropriate treatment modality.

Conflicts of Interest

The authors have no potential conflicts of interest.

Funding

None.

Author Contributions

Conceptualization: Neil Robert O’Morain, Helen O’Donovan, Caroline Conlon, Eileen Shannon

Data curation: NROM, HOD, CC, ES

Formal analysis: NROM, HOD, CC, ES

Investigation: NROM, HOD, CC, ES

Methodology: NROM, HOD, CC, ES, Diarmuid Manning, Eoin Slattery

Project administration: NROM, HOD, CC, ES

Resources: NROM, HOD, CC, ES

Software: NROM, HOD, CC, ES

Supervision: NROM, HOD, CC, ES, DM, ES

Validation: NROM, HOD, CC, ES

Visualization: NROM, HOD, CC, ES

Writing-original draft: NROM, HOD, CC, ES

Writing-review\&editing: NROM, HOD, CC, ES, DM, ES

\section{ORCID}

Neil Robert O’Morain:

Helen O’Donovan:

Caroline Conlon:

Eileen Shannon:

Diarmuid Manning:

Eoin Slattery:

https://orcid.org/0000-0003-2542-9455
https://orcid.org/0000-0003-4868-0255
https://orcid.org/0000-0002-9211-3488
https://orcid.org/0000-0003-2391-8757
https://orcid.org/0000-0002-2900-4246
https://orcid.org/0000-0003-1543-1586

\section{REFERENCES}

1. Suit PF, Petras RE, Bauer TW, Petrini JL Jr. Gastric antral vascular ectasia. A histologic and morphometric study of "the watermelon stomach". Am J Surg Pathol 1987;11:750-757.

2. Gilliam JH 3rd, Geisinger KR, Wu WC, Weidner N, Richter JE. Endoscopic biopsy is diagnostic in gastric antral vascular ectasia. The "watermelon stomach". Dig Dis Sci 1989;34:885-888.

3. Lewis TD, Laufer I, Goodacre RL. Arteriovenous malformation of the stomach. Radiologic and endoscopic features. Am J Dig Dis 1978;23:467-471.

4. Jabbari M, Cherry R, Lough JO, Daly DS, Kinnear DG, Goresky CA.
Gastric antral vascular ectasia: the watermelon stomach. Gastroenterology 1984;87:1165-1170.

5. Petrini JL Jr, Johnston JH. Heat probe treatment for antral vascular ectasia. Gastrointest Endosc 1989;35:324-328.

6. Binmoeller KF, Katon RM. Bipolar electrocoagulation for watermelon stomach. Gastrointest Endosc 1990;36:399-402.

7. Rose JD. Endoscopic injection of alcohol for bleeding from gastroduodenal vascular anomalies. Br Med J (Clin Res Ed) 1987;295:93-94.

8. Sargeant IR, Loizou LA, Rampton D, Tulloch M, Bown SG. Laser ablation of upper gastrointestinal vascular ectasias: long term results. Gut 1993;34:470-475.

9. Wahab PJ, Mulder CJ, den Hartog G, Thies JE. Argon plasma coagulation in flexible gastrointestinal endoscopy: pilot experiences. Endoscopy 1997;29:176-181.

10. Herrera S, Bordas JM, Llach J, et al. The beneficial effects of argon plasma coagulation in the management of different types of gastric vascular ectasia lesions in patients admitted for GI hemorrhage. Gastrointest Endosc 2008;68:440-446.

11. Kwan V, Bourke MJ, Williams SJ, et al. Argon plasma coagulation in the management of symptomatic gastrointestinal vascular lesions: experience in 100 consecutive patients with long-term follow-up. Am J Gastroenterol 2006;101:58-63.

12. Chaves DM, Sakai P, Oliveira CV, Cheng S, Ishioka S. Watermelon stomach: clinical aspects and treatment with argon plasma coagulation. Arq Gastroenterol 2006;43:191-195.

13. Yusoff I, Brennan F, Ormonde D, Laurence B. Argon plasma coagulation for treatment of watermelon stomach. Endoscopy 2002;34:407-410.

14. Boltin D, Gingold-Belfer R, Lichtenstein L, Levi Z, Niv Y. Long-term treatment outcome of patients with gastric vascular ectasia treated with argon plasma coagulation. Eur J Gastroenterol Hepatol 2014;26:588-593.

15. Nakamura S, Mitsunaga A, Konishi H, Oi I, Shiratori K, Suzuki S. Longterm follow up of gastric antral vascular ectasia treated by argon plasma coagulation. Dig Endosc 2006;18:128-133.

16. Garg S, Aslam B, Nickl N. Endoscopic resolution and recurrence of gastric antral vascular ectasia after serial treatment with argon plasma coagulation. World J Gastrointest Endosc 2017;9:263-266.

17. Jana T, Thosani N, Fallon MB, Dupont AW, Ertan A. Radiofrequency ablation for treatment of refractory gastric antral vascular ectasia (with video). Endosc Int Open 2015;3:E125-E127.

18. Magee C, Lipman G, Alzoubaidi D, et al. Radiofrequency ablation for patients with refractory symptomatic anaemia secondary to gastric antral vascular ectasia. United European Gastroenterol J 2019;7:217-224.

19. Wells CD, Harrison ME, Gurudu SR, et al. Treatment of gastric antral vascular ectasia (watermelon stomach) with endoscopic band ligation. Gastrointest Endosc 2008;68:231-236.

20. Keohane J, Berro W, Harewood GC, Murray FE, Patchett SE. Band ligation of gastric antral vascular ectasia is a safe and effective endoscopic treatment. Dig Endosc 2013;25:392-396.

21. Zepeda-Gómez S, Sultanian R, Teshima C, Sandha G, Van Zanten S, Montano-Loza AJ. Gastric antral vascular ectasia: a prospective study of treatment with endoscopic band ligation. Endoscopy 2015;47:538-540.

22. Sato T, Yamazaki K, Akaike J. Endoscopic band ligation versus argon plasma coagulation for gastric antral vascular ectasia associated with liver diseases. Dig Endosc 2012;24:237-242.

23. Eccles J, Falk V, Montano-Loza AJ, Zepeda-Gómez S. Long-term follow-up in patients with gastric antral vascular ectasia (GAVE) after treatment with endoscopic band ligation (EBL). Endosc Int Open 2019;7:E1624-E1629.

24. Polski JM, Brunt EM, Saeed ZA. Chronology of histological changes after band ligation of esophageal varices in humans. Endoscopy 2001;33:443-447. 